Revista Tecné, Episteme y Didaxis: TED. Año 2014, Número Extraordinario. ISSN Impreso: 0121-3814, ISSN web: 2323-0126

Memorias, Sexto Congreso Internacional sobre Formación de Profesores de Ciencias. 08 al 10 de octubre de 2014, Bogotá

\title{
Physics Tracker: Una implementación didáctica para la presentación del tema tiro parabólico en bachillerato
}

Méndez, Germán.' Rodríguez, Stella

Categoría 1. Reflexiones y experiencias desde la innovación en el aula.

\section{Resumen}

A continuación se presenta una secuencia de didáctica para grado décimo en el colegio Kapeirot, en el que se hace uso de la metodología de enseñanza por investigación orientada, para la temática de tiro parabólico con juegos cotidianos en los que se evidencia una trayectoria parabólica, la cual es analizada con el software libre Physics tracker, para realizar un posterior análisis cinemático.

\section{Palabras clave}

Movimiento en dos dimensiones, investigación orientada, enseñanza de la física, physics tracker

\section{Introducción}

Los jóvenes actualmente ven en las ciencias "puras" un tema monótono alejado del mundo real que sólo está destinado a mentes brillantes detrás de un laboratorio (Pérez G. 2005) y por ende no es necesario para ellos aprender las temáticas científicas, por lo que no es raro escuchar en clase a los estudiantes decir: "¿para que necesito saber física si yo no voy a estudiar eso?" Pues bien, la respuesta de algunos docentes es porque hace parte del currículo que se establece desde los estándares educativos del país y lo tienen que aprender, esta visión da aún más argumentos a los estudiantes para que muestren apatía por el estudio de las ciencias naturales. Sin embargo cuando se trata de entender las juventudes actuales, se nota cómo ellos están inmersos en un mundo de aplicaciones tecnológicas y que los docentes muchas veces no hacemos uso de las mismas en clase sino por el contrario las cohibimos, cuando pueden ser la

\footnotetext{
1 Colegio Kapeirot, gamendezm114@gmail.com
} 
Revista Tecné, Episteme y Didaxis: TED. Año 2014, Número Extraordinario. ISSN Impreso: 0121-3814, ISSN web: 2323-0126

Memorias, Sexto Congreso Internacional sobre Formación de Profesores de Ciencias. 08 al 10 de octubre de 2014, Bogotá

herramienta para atraerlos a un estudio científico. Por otro lado, los problemas de la enseñanza de la física ha radicado en que sus temáticas son presentadas como trabajos ya terminados en el mundo de la ciencia, por ejemplo la mayoría de textos muestran un fragmento de quién inventó la teoría, luego muestra unas ecuaciones y problemas de aplicación en los que se utilizan las fórmulas mencionadas, terminando así la temática con unos ejercicios de práctica. El estudiante al ver estas presentaciones se convence de que la física es seguir fórmulas en las que se reemplazan por números que da un enunciado y finalizar, cuando ello deja de lado un factor importante de la física y es que las teorías físicas se enmarcan dentro de un límite de posibilidades de aplicabilidad.

Por ende lo que se busca en este trabajo es una presentación para que el estudiante desarrolle un proceso de (re) construcción de las teorías físicas, como es el movimiento en dos dimensiones (en adelante 2D), inspirado en las ayudas tecnológicas y la indagación desde una discusión de interés, en este caso juegos cotidianos en los que se evidencie una trayectoria parabólica.

\section{¿Cuál podría ser la presentación del movimiento en 2D, para estudiantes de bachillerato?}

Este trabajo inicia con la pregunta: ¿̇qué tipos de deportes evidencian una trayectoria parabólica? Esta pregunta abierta lo que busca es dar sentido a un estudio cinemático de una situación física real (hasta este punto los estudiantes ya habían visto movimiento rectilíneo y caída libre), además que el estudiante se forme una primera idea motivadora al tema de 2D. Con la idea motivadora, se da paso a un estudio cualitativo del deporte escogido, que si bien, fueron diversos deportes, es donde una orientación adecuada del docente fortalece: la estructura matemática de vectores, la compresión de lo que se modela como un espacio medible en dos direcciones diferentes para un mismo evento, pues es el estudiante se ve inmerso directa o indirectamente en saber: ¿̇cómo mido la altura de mi objeto? ¿cómo saber la distancia que alcanzará mi objeto? ¿̇cómo controlar el lanzamiento para repetir mis mediciones y saber si son correctas? Estas preguntas el estudiante las puede mediar a través del uso del programa Physics tracker (en adelante PT); para ello es necesario tener algunas condiciones iniciales del evento y a la vez el uso de aparatos tecnológicos como una cámara digital con opción de video y/o los celulares "inteligentes" que hoy día poseen algunos estudiantes. 
Revista Tecné, Episteme y Didaxis: TED. Año 2014, Número Extraordinario. ISSN Impreso: 0121-3814, ISSN web: 2323-0126

Memorias, Sexto Congreso Internacional sobre Formación de Profesores de Ciencias. 08 al 10 de octubre de 2014, Bogotá

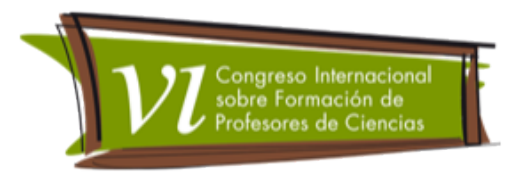

En suma todo lo anterior se presenta como una clase diferente, en la que el estudiante interactúa con aparatos tecnológicos y comienza a familiarizarse con el quehacer científico, desde la indagación y el planteamiento de hipótesis para una posible solución, donde el docente juega un papel importante en la medida que comienza a cerrar las posibilidades y enfocar el estudio al movimiento en 2D, en especial con la búsqueda de relaciones de posiciones y tiempos, para que logre llegar a relaciones secundarias como velocidad y aceleración.

¿Qué puede esperarse de los estudiantes conceptualmente, en el análisis de un movimiento en dos dimensiones y/ó movimiento parabólico?

En mención al ítem anterior el estudiante esta en el desafío de ordenar unos pasos para conseguir unas medidas que le sirvan para su posterior análisis y realice un contraste teóricamente para corroborar o confrontar resultados. La formulación de hipótesis se hace inminente por parte de los estudiantes, entre ellas: ¿̇qué pasa en el punto más alto del objeto? ¿̇cómo asegurar el mismo punto de lanzamiento? ¿̇qué necesito para medir? Todas las preguntas se conectan en un concepto que en ocasiones no se hace alusión en la temática del movimiento parabólico y es el marco de referencia. Lo esencial es entonces que se manifiesten las preguntas de discusión, las hipótesis y la puesta en práctica de estrategias de resolución, donde el docente orienta a que los estudiantes desarrollen de manera adecuada y acertada los objetivos que se proponen, como el de un lenguaje matemático vectorial básico y un argumento sólido a través de la evidencia y de los conceptos que previamente ya se habían dictado en el curso. Es entonces cuando el estudiante podría encontrarse con relaciones de temáticas vistas con anterioridad y darse cuenta de un hilo conductor en el la construcción del movimiento parabólico, que es la unión del movimiento rectilíneo uniforme y caída libre, además de comenzar a intuir el concepto de marcos de referencia y el concepto de cantidades vectoriales.

\section{Y los estudiantes ¿qué hacen con el programa Physics Tracker? Y ¿a qué llegaron?}

El estudiante en clase realiza la toma de diferentes videos del deporte escogido en que se evidencia la trayectoria parabólica, para luego obtener datos mediante el programa PT, software que es explicado en términos que el "algoritmo" que se presenta no sea un paso a paso para el estudiante, sino por el contrario, las indicciones son genéricas para que llamen la atención del estudiante y con ello logre encontrar relaciones en el evento escogido. Éstas 
Revista Tecné, Episteme y Didaxis: TED. Año 2014, Número Extraordinario. ISSN Impreso: 0121-3814, ISSN web: 2323-0126

Memorias, Sexto Congreso Internacional sobre Formación de Profesores de Ciencias. 08 al 10 de octubre de 2014, Bogotá

relaciones hacen parte del análisis de gráficas, como se muestran en la siguiente imagen de algunos de los videos realizados por estudiantes del colegio Kapeirot de grado décimo:

Gráfica 1: Estudiantes de grado décimo del colégio Kapeirot.
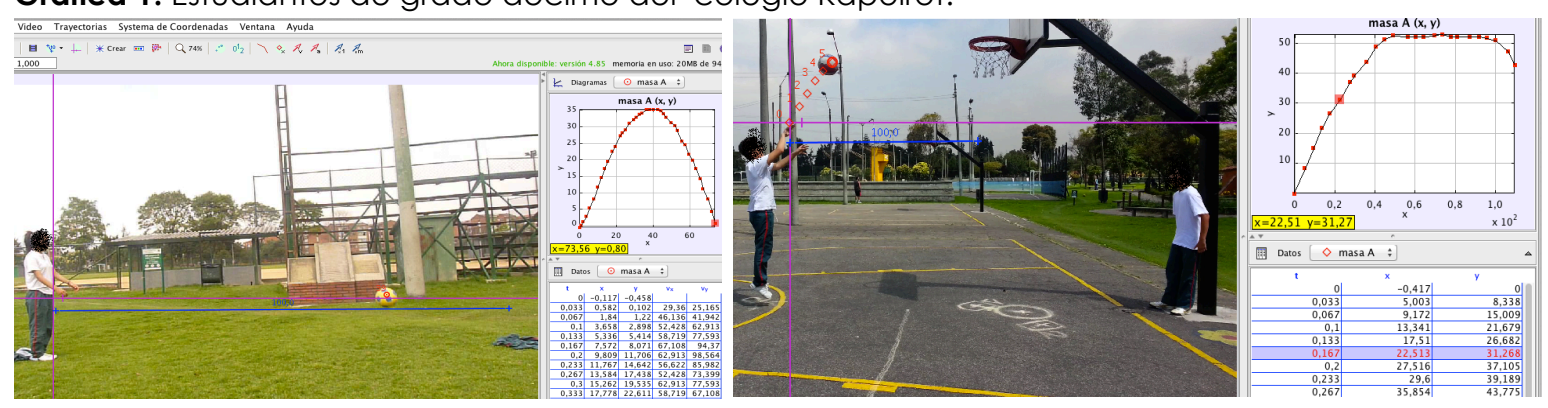

\section{Sobre la gráfica 1}

La gráfica 1, evidencia de manera matemática la trayectoria parabólica y semiparabólica, de dos deportes claramente diferenciados, por un lado el lanzamiento de voleibol (izquierda) y lanzamiento de baloncesto (derecha), de modo que la mayoría de los estudiantes mostro en sus laboratorios, que el movimiento parabólico es una descripción del cambio de posición en el eje equis y en el eje ye, que al comparar sus graficas, con lo que muestra la teoría de textos e internet, asemejan que en el eje $x$ se tiene un m.r.u. y en del eje ye se tiene caída libre, que los une una variable que es el tiempo. Por tanto los estudiantes de manera implícita encuentran que el movimiento parabólico es la superposición de dos movimientos. Por otro lado el manejo de las cantidades vectoriales para los estudiantes se hizo amena, dado que al momento de darle las condiciones iniciales al programa, se tuvo que medir de forma experimental la distancia en equis e ye, como se evidencia en la gráfica 1 , donde la estudiante (izquierda) uso el buzo de la sudadera como un marco referencial, mientras el estudiante (derecha) uso tiza sobre el lugar de lanzamiento, así en los análisis posteriores del informe pedido a los estudiantes se vio como la gran mayoría definió desde la experiencia los valores de la velocidad en equis e ye, destacando que cada una de ellas correspondía a fenómenos ya vistos, lo que mejora una compresión de la cinemática, debido a que el estudiante, por un lado recuerda la temática vista y retroalimenta conceptos base de un movimiento 2D, como el de cantidades vectoriales, que no son mas el manejo de trigonometría básica. 
Revista Tecné, Episteme y Didaxis: TED. Año 2014, Número Extraordinario. ISSN Impreso: 0121-3814, ISSN web: 2323-0126

Memorias, Sexto Congreso Internacional sobre Formación de Profesores de Ciencias. 08 al 10 de octubre de 2014, Bogotá

\section{Conclusiones}

La realización de la temática del movimiento parabólico para estudiantes de grado décimo del colegio Kapeirot a través de deportes que evidencien la trayectoria de una parábola, mostro una adquisición del concepto de vectores para situaciones físicas en la modelación de eventos en 2D sobre un plano cartesiano, haciendo uso de las relaciones trigonométricas para la obtención de los valores de la velocidad por componentes.

El método de enseñanza por investigación orientada, mejoro la relación enseñanza-aprendizaje en el tema de movimiento parabólico porque partió de una situación abierta y llamativa hacia el estudiante, para luego confrontar diferentes puntos de vista: los desplazamientos a medir, las condiciones iniciales en la toma de datos, la contrastación teórica experimental del evento evidenciado y así retomar temáticas vistas orientadas por parte del docente hacia el movimiento parabólico como la superposición de dos estados físicos uno del eje equis (movimiento rectilíneo uniforme) y el otro en el eje ye (caída libre).

Los estudiantes se ven atraídos por el manejo del software, en los que ven que la medición de eventos físicos no solo debe remitirse a una regla o elementos caseros, sino que la tecnología presta una mejora en implementos de medición y que son mas precisos a la hora de comprobar datos, además de ello Physics Traker, realiza toma de datos reales y no simulados, que en ocasiones la simulación deja de lado aspectos físicos relevantes como la resistencia del aire, entre otros, mientras que un video reproduce lo que sucede realmente en la medición física, mostrando al estudiante las condiciones que deben colocarse para que se cumpla el objetivo planteado a lo largo de un movimiento en 2D, como lo es el movimiento parabólico.

\section{Referencias bibliográficas}

Bohigas X, Jaén X \& Navell M (2003) Applets en la enseñanza de la física, Innovaciones didácticas, Universidad Polictecnica de Catalunia: España.

Chacón C. (2008) Problemáticas fundamentales de la formación en física básica Tecné, episteme y didaxis Bogotá: Colombia pág.131-140.

Cohen, L., \& Manion L. (1990).Métodos de investigación educativa. España: Editorial La Muralla, S.A. 
Revista Tecné, Episteme y Didaxis: TED. Año 2014, Número Extraordinario. ISSN Impreso: 0121-3814, ISSN web: 2323-0126

Memorias, Sexto Congreso Internacional sobre Formación de Profesores de Ciencias. 08 al 10 de octubre de 2014, Bogotá

Colegio Kapeirot pagina web:

http://colegiokapeirot.edu.co

Dolores C., AlarconG \& Albarrán D (2002). Concepciones alternativas sobre las gráficas del movimiento: el caso de la velocidad y la trayectoria. Relime Vol. 5 pág. 225-250.

Furió, C., y Guisasola, J. (1998).Dificultades de aprendizaje de los conceptos de carga y de campo eléctrico en estudiantes de bachillerato y universidad, Enseñanza de las Ciencias, Vol 16, No 1, pág.131-146.

Gil-Pérez, D., Macedo, B., Martínez Torregosa, J., Sigifredo C., Valdés, P., \& Vilches, A., (2005). ¿Cómo promover el interés por la cultura científica?, una propuesta fundamentada para la educación científica de jóvenes de 15 a 18 años. Santiago de Chile: Andros Impresores.

Gómez M, Castañeda L, Vela J, Álvarez s \& Navarrete J. Uso de laboratorios virtuales en la Enseñanza- Aprendizaje de la física. Entre la ciencia e ingeniería Pereira Colombia pág. 83-93.

Organista O., Gómez V., Jaimes D \& Rodríguez J (2007) Una idea profunda en la compresión del mundo físico: el principio de superposición de estados Latin American Journal of Physical Education. Vol 1.

Pacca J, Pacca L y Villani A (1996) Un curso de actualización y cambios conceptuales en profesores de física. Universidad de Sao Paulo. Sao Paulo: Brasil.

Página web del programa Physic Tracker: http://www.cabrillo.edu/ dbrown/tracker/

Pozo J. (1987) La historia se repite: las concepciones espontaneas sobre el movimiento y la gravedad. Universidad autónoma de Madrid, Madrid: España pág. 69-87.

Rodríguez J, (s.f.) conferencia: ¿̇ónde esta la física? Universidad de Boyacá 